Mitteilungen aus den chemiscnen Instituten der Universitäten Greifswald und Marburg. .

\title{
Spektrochemische Untersuchungen:
}

von K. $v$. Auwers.

(Eingelaufen am 25. November 1914.)

\section{Einleitung.}

Die Beschäftigung mit der Frage, wieweit sich die Spektrochemie zur Feststellung von Gleichgewichsverhältnissen zwischen Keto- und Enolformen verwerten lasse ${ }^{1}$, führte mich dazu, bestimmte Reihen sauerstoffhaltiger Benzolderivate etwas genauer optisch zu untersuchen. Ebenso erforderte das Studium der Cumaranongruppe $^{2}$ ) spektrochemische Versuche mit Cumaronderivaten und ähnlich gebauten Benzolabkömmlingen. Dabei wurden Beobachtungen gemacht, die auf bisher nicht bekannte gesetzmäßige Beziehungen zwischen chemischer Konstitution und optischem Verhalten aromatischer Substanzen hindeuteten und eine weitere Ausdehnung dieser Versuche nötig machten. Wenn auch bei der schier unerschöpflichen Mannigfaltigkeit, die auf dem Gebiete der aromatischen Körpergruppen herrscht, von einem $A b$ schluß dieser Untersuchungen nicht die Rede sein kann, läßt doch das bisher gesammelte Material einige Regelmäbigkeiten schon so klar hervortreten, dab ihre allgemeine Gültigkeit innerhalb bestimmter Grenzen als bewiesen angesehen werden darf.

Ich veröffentliche daher in den folgenden beiden Arbeiten einige Beiträge zur Spektrochemie der Benzolderivate und der heterocyclischen Verbindungen. Das zugrunde liegende Material ist zum allergröBten Teil neu.

1) Ber. d. d. chem. Ges. 44, 3514, 3525 (1911).

2) Ber. d. d. chem. Ges. 43, 2192 (1910); 45, 976 (1912); diese Annalen 393, 338 (1912); Ber. d. d. chem. Ges. 47, 2334 (1914). 
Wo fremde Beobachtungen herangezogen wurden, sind sie zuvor neu berechnet worden, wobei die genauen Molekulargewichte und die Kisenlohrschen Atomrefraktionen benutzt und erforderlichenfalls die Dichteangaben auf die Basis Wasser von $4^{0}$ umgerechnet wurden. Die Originalzahlen dieser fremden and eigener, bereits früher veröffentlichter Beobachtungen, die mit verwertet wurden, können an den angęführten Literaturstellen nachgesehen werden und zur Kontrolle dienen.

Im allgemeinen Teil jeder Arbeit, der einen Überblick über die Ergebnisse und deren Besprechung enthält, werden, wie früher, nur die Exallationen der spezifischen Refraktion und Dispersion, also die E $\mathrm{E}$-Werte, der einzelnen Verbindungen aufgeführt, da sie in erster Linie für die Charakteristik und für die Zwecke der Vergleichung in Betracht kommen. Außerdem sind in eine Reihe von Tabellen auch die auf die Temperatur von $20^{\circ}$ umgerechneten Dichten und Brechungsindizes für Natriumlicht aufgenommen worden, denn die Zusammenstellung solcher vergleichbarer Konstanten kann in mancher Hinsicht nützlich sein. Nur wenn die Bestimmungen von Dichte und Brechungsinảex bei höherer Temperatur ausgeführt wurden, ist von einer Umrechnung abgesehen worden, da die erhaltenen Zahlenwerte nicht genügend sicher sein würden. Alle unmittelbaren Beobachtungsdaten sind in den experimentellen Teilen zusammengestellt, wo sich auch die nötigen Angaben über Darstellung und Reinigung der untersuchten Präparate und die Beschreibung der noch nicht bekannten Verbindungen findet. Physikalische Daten, die von früheren Beobachtungen herrühren, sind angeführt, soweit dies erforderlich schien. Hierbei sind Werte, die aus den Originalzahlen durch Umrechnung erhalten wurden, durch ein Sternchen $\left({ }^{*}\right)$ gekennzeichnet. Wo Angaben über $\mathrm{n}_{\gamma}$ und $\mathrm{M}_{\gamma}-\mathrm{M}_{\alpha}$ fehlen, konnte die Linie $\mathrm{H}_{\gamma}$ wegen zu starker Färbung der Präparate oder aus anderen Gründen nicht abgelesen werden. Ein bei- 
gesetztes Fragezeichen bedeutet, daB die Ablesung der betreffenden Linie unsicher war. Sämtliche Dichtebestimmungen sind auf den luftleeren Raum berechnet. Die üblichen Vorsichtsmaßregeln, die namentlich bei leicht polymerisierbaren oder leicht oxydierbaren Substanzen eingehalten wurden, brauchen nicht im einzelnen aufgezählt zn werden.

Am Schluß der ganzen Mitteilung sind die wesentlichen Ergebnisse der beiden Arbeiten, soweit sie Tatsachen betreffen, nochmals kurz zusammengestellt.

Die Untersuchungen sind mit großen Unterbrechungen im Laufe der letzten vier Jahre ausgeführt worden. Als Nitarbeiter haben daran hauptsächlich die Herren UDr. A. Boennecke, F. Krollpfeiffer and G. Peters teilgenommen; bei einigen Verbindungen auch Hr. Dr. E. Lange. Allen diesen bewährten Helfern sage ich herzlichen Dank.

\section{Zur Spektrochemie der Benzolderivate.}

Nach den zurzeit herrschenden Anschaungen wird das spektrochemische Verhalten von Benzolderivaten im allgemeinen durch zwei, von $\mathrm{Brühl^{1 }}$ ) aufgestellte, Grundregeln bestimmt. Erstens sollen die nach Kekulé im Benzolring vorhandenen drei Doppelbindungen ein in optischer Beziehung "neutral konjugiertes" System bilden, so daß Exaltationen, die sonst die Folge der konjugierten Lage von Doppelbindungen sind, in der Regel nicht auftreten. Lagert sich aber an den Benzolkern eine ungesättigte Gruppe irgendwelcher Art an, deren Doppelbindung oder Nebenvalenzen mit einer Doppelbindang des Ringes eine Konjugation bilden können, dann entsteht dadurch ein "aktuell konjugiertes" System, das die

') Ber. d. d. ch m. Ges. 40, 878 (1907). 